\title{
Impact de la crise pandémique sur la Suply Chain Management au Maroc : cas de port de Tanger Med
}

\author{
Achemrah Younès \\ Université Abdelmalek Essaadi, Tétouan, Maroc
}

\begin{abstract}
Résumé : Dans un environnement en évolution continue, les entreprises et les économies sont de plus en plus confrontées à une concurrence internationale très agressive, à des changements technologiques incessants et aux attentes croissantes des clients. Cette situation sera bouleversée au cours de l'année 2020 où le monde se retrouve en confrontation face à une crise sanitaire sans précédent marquée par la propagation du Coronavirus. Par conséquent, toutes les activités économiques se sont impactées à cause de la fermeture des frontières nationales et l'arrêt de la production dans le monde, notamment au Maroc où la crise pandémique a profondément touché des différents domaines d'activités stratégiques du pays, particulièrement, le secteur de la logistique et du transport, sachant que ce secteur est considéré comme l'un des créneaux les plus importants pour l'économie du Maroc. Cet article s'intéresse à l'analyse de l'impact de cette crise sanitaire survenue de manière inattendue sur la Suply Chain Management au Maroc et plus précisément au niveau de l'activité portuaire de port Tanger Med.
\end{abstract}

Mots-clés : Environnement, entreprise, économie, concurrence, client, crise.

\section{Introduction}

Sous l'effet de la mondialisation et la révolution technologique, l'évolution de la Suply Chain Managment s'est produite rapidement depuis plus de deux décennies. En effet, l'accélération de l'ouverture économique initiée par le mouvement de la libéralisation, le rôle croissant des investissements directs étrangers dans les économies nationales, le climat instable des marchés et les changements de l'environnement économique international liés à la concurrence a poussé les acteurs économiques à modifier leurs comportements notamment les entreprises au niveau de leurs stratégies. Il en résulte des stratégies de concentration sur le client perçues comme une reformulation des politiques, des plans et des stratégies déjà existantes face à une ère de compétition mondiale. Le nouveau paysage économique au niveau international est à l'origine de nouvelles logiques industrielles et commerciales. Dans cette mouvance, le processus de production doit désormais être axé sur le client final. Une 
entreprise qui cherche à conquérir les marchés pour réussir à commercialiser ses produits dans un environnement de forte concurrence est appelée à assurer les fonctions d'approvisionnement, de production, de distribution et de commercialisation. Ces fonctions forment la chaine logistique ou Suply Chain, et la réussite de cette chaine oblige l'entreprise à gérer de manière optimale une intégration fonctionnelle impliquant l'imbrication entre les quatre fonctions tout en tenant compte des autres fonctions d'aide et de soutien de l'organisation telles que le capital humain, les ressources financières, etc. Ces facteurs contribuent donc à réussir le management de la chaine logistique. Cette dernière se retrouve en face du défi à relever dans les circonstances nouvelles à savoir celui de l'exécution de la production de l'entreprise, afin de satisfaire la demande du client et permettre à l'entreprise de réaliser ses objectifs de chiffre d'affaire et de gains.

Les trois principaux leviers qui constituent un challenge pour l'organisation, et sur lesquels il est possible d'agir pour améliorer la performance de toute entreprise sont : la création de valeur, la productivité des moyens et ressources utilisées par l'entreprise, la satisfaction du client grâce à la connaissance de ses besoins). Pour chacun de ces leviers, il est nécessaire de s'assurer de la disponibilité des ressources à mobiliser et de mesurer l'impact des décisions prises à l'aide d'indicateurs de performance et d'inscrire cette démarche logistique dans la durée comme partie intégrante de la stratégie de l'entreprise.

La montée du concept de la Suply Chain Management dans les médias et le monde des affaires coïncide avec une période de fermeture des frontières nationales de la plus part des pays du monde suite à la crise sanitaire sans précédent relative à la propagation de la pandémie Covid 19. Cette crise internationale a impactée toutes les économies mondiales y comprises celle du Maroc à des niveaux différents. De nombreux secteurs économiques et stratégiques du Royaume ont subi les conséquences néfastes de la fermeture totale du pays et de l'arrêt de l'activité économique, particulièrement, le secteur de la logistique et du transport.

Cet article sera divisé en deux parties. La première partie sera consacrée à présenter l'aspect théorique du concept de la Suply Chain de Management, de son apparition, de son évolution et de ses enjeux sur l'activité logistique et portuaire au Maroc à l'ère de la pandémie. L'impact de la crise pandémique sur le processus de la Suply Chain de Management au Maroc avec l'étude de cas du port Tanger Med avant et après l'apparition du virus Covid-19 fera l'objet de la deuxième partie. Le but recherché de cet article est de répondre à la question suivante : quel est l'effet provoqué par la crise sanitaire mondiale survenue au cours de l'année 2020 sur la Suply Chain de Management au Maroc notamment sur l'activité au niveau du port Tanger Med.

Pour élaborer cet article, on a opté pour une étude axée sur une méthodologie descriptive et analytique consistant à décrire l'évolution du concept de la Suply Chain de Management de point de vue théorique. Ensuite, la recherche sera étendue à l'analyse de l'évaluation de l'impact de la crise sanitaire 
sur la Suply Chain de Management au niveau de l'activité du port Tanger Med. Cette évaluation des répercussions attendues de la crise pandémique est une étape nécessaire pour remédier et traverser l'impasse de la situation actuelle tout en adoptant des mesures rapides pour limiter les pertes économiques et opter pour une vision stratégique de la gestion des crises face aux risques qui pourraient survenir dans l'avenir. L'option stratégique pour mieux gérer et réussir à confronter les fléaux et les crises du futur est perçue comme vitale pour le social, l'entreprise et l'administration mais aussi pour la croissance économique en général.

\section{Emergence et évolution de la Suply Chain Management}

\subsection{Emergence de la SCM}

L'histoire nous apprend que La Supply Chain Management est née d'abord dans l'armée. Il émane de l'officier en charge de la « logis » qui s'occupe des troupes pendant la bataille, mais est également responsable de toute l'organisation en dehors de la bataille. Les guerres mettent la logistique en pratique en termes de réflexion stratégique. Ainsi, la Supply Chain Management est devenue un facteur déterminant dans l'organisation des opérations militaires.

Ensuite, dans l'industrie automobile, la Supply Chain Management a d'abord été utilisée pour gérer au mieux le stockage et l'entreposage des pièces détachées et des produits.

Avec la révolution industrielle porteuse d'évolutions technologiques majeures, la SCM s'est vu accélérer son développement avec une gestion optimale du trafic.

Durant les années 1980 et 1990, la Supply Chain Management était en phase de croissance et est devenue un élément central de l'entreprise. On commence à voir une organisation simple, mais dans une démarche d'optimisation de l'efficacité et de maîtrise des coûts.

La SCM est généralement associé aux activités du transport responsable de la livraison et du stockage des marchandises. C'est une activité qui vise à gérer la circulation physique des marchandises entre les acteurs économiques et les différents services de l'entreprise.

.ASLOG $2^{1}$, association qui regroupe les acteurs de la Supply chain, définit la logistique comme "l'art et la manière de fournir des produits au bon moment, au bon endroit, au moindre coût et avec la meilleure qualité".

Pour cela, le personnel logistique a pour mission de coordonner avec tous les intervenants internes et externes avant, pendant et après la production. Cela implique la gestion des fournisseurs et des matières

\footnotetext{
${ }^{1}$ www.revues.imist.ma.com
} 
premières, puis la gestion des stocks et des entrepôts, et enfin la préparation des commandes, la gestion des processus de distribution et des commandes d'expédition.

\subsection{L'évolution de la Suply Chain de Management}

Vers la fin des années 90, la Gestion de Chaines Logistiques ou Supply Chain Management (SCM) commence à faire son apparition à la fois dans les médias, le monde de l'entreprise et la chaine de production. Ce concept se définit comme « un ensemble des taches et des acteurs agissant sur les flux physiques et informationnels, pour assurer la transformation de produit de l'état de matière première à celui de produit fini commercialisé auprès du client. Le client est donc au centre de cette gestion, il est à la fois le but et la cible. Pour cela, les outils et les méthodes pratiqués dans ce processus consistent à recentrer l'organisation, essentiellement sur le client, ses besoins et ses désirs.

La chaîne logistique peut être vue également comme un ensemble (de personnes, d'actions, de ressources ou d'opérations), un système, un enchaînement de processus, un réseau (d'organisations ou d'entreprises) ou encore comme une structure réticulaire dynamique à l'origine des flux. Elle peut même devenir un mode d'analyse. La SCM est un mode de gestion, de coordination, d'échange voire une philosophie managériale de cette chaîne.

Le développement de la Suply Chain de Management à la fin des années 90 coïncide avec le développement rapide des Nouvelles Technologies de l'Information et de la Communication et la montée de la mondialisation. Ce progrès constitue une révolution technologique qui bouleversera par la suite les habitudes et les pratiques des acteurs économiques. Ainsi, l'ère de l'Information fournit un accès nouveau et sans restrictions à l'aspect "lieu" des affaires. Les technologies d'entreposage ont drastiquement changé avec l'introduction de l'informatique depuis les bureaux jusqu'aux élévateurs (stockage automatisé...). Les technologies nouvelles facilitent la communication commerciale et technique entre les partenaires industriels dans un environnement d'une économie mondialisée. Cette évolution des organisations industrielles reste basée sur le modèle d'intégration du système de production, dont les frontières dépassent désormais celles de l'entreprise pour atteindre celle d'une entreprise résultant d'un partenariat diversifié et durable entre de nombreux acteurs industriels.

\subsection{Le concept de Suply Chain Managment}

\subsubsection{Définition}

La chaine logistique ou la Suply Chain Management peut être définie comme un ensemble (de personnes, d'actions, de ressources ou d'opérations), un système, un enchaînement de processus, un réseau (d'organisations ou d'entreprises) ou encore comme une structure réticulaire dynamique à l'origine des flux. Elle peut même devenir un mode d'analyse. La SCM est aussi un mode de gestion, de coordination, d'échange voire une philosophie managériale de cette chaine. 
La Suply Chain de Management a été différemment interprétée par de nombreux auteurs. De multiples définitions existent.

Pour Cooper et Ali (1997), la SCM est une stratégie qui tend vers une gestion intégrée de l'ensemble des flux du canal de distributions des fournisseurs à l'utilisation finale ${ }^{2}$. Cette définition signifie la nécessité de l'intégration des flux et des acteurs.

Pour TAN et AL (1998), la SCM comporte le management des approvisionnements depuis la matière première de base jusqu'à la mise à disposition du produit final (et éventuellement le recyclage). La SCM se focalise sur l'avantage compétitif et les modalités liées à l'utilisation par firmes des processus, technologies et capacités gérées par les fournisseurs. Il représente une technologie intraorganisationnelle traditionnelle des partenaires commerciaux vers un objectif commun d'optimisation et d'efficacité3.

Cette définition met l'accent sur la nécessité d'une vision partagée intra et inter-organisationnelle permettant de créer la valeur relationnelle.

Pour Morana et Paché (2003), la Suply Chain de Management est un ensemble d'activités et de ressources dédiés à la circulation de produits et matières.

Detchessahar et al (2003), la SCM est définie comme un système de circulation physique des produits donnant accès aux modèles d'affaires.

Halley (2004), la SCM se définit comme un réseau d'entreprises en interaction dont l'objectif est de livrer un produit ou un service à l'utilisateur final en coordonnant les activités associées au mouvement des biens de la matière première jusqu'à la livraison du produit fini par des combinaisons efficaces de ressources qui contribuent à la création et à la livraison de la valeur. Cette suply se fait selon trois niveaux : planification conjointe et coordination pour la gestion des stocks, le processus de commande, la réception et la manutention, la préparation des commandes, l'expédition et la livraison ( logistique administrative), l'intégration des processus clés pour les relations avec les clients et le service, la gestion de la demande, l'exécution des commandes, la gestion des flux, le MRP (logistique tactique), et l'alignement stratégique pour établir des buts et objectifs communs, des relations adaptées au contexte, un partage des ressources, des mesures intégrées de la performance ( logistique stratégique).

\footnotetext{
${ }^{2}$ LOGISTIQUE MAGAZINE, Octobre2007, №221, p72.
}

${ }^{3}$ LOGISTIQUE MAGAZINE, Octobre2007, №221, p72. 
Chopra et Mindel (2004) considèrent que la SCM représente un management des flux entre et parmi les niveaux de la Supply dans le but de maximiser la profitabilité totale de la Supply Chain ${ }^{4}$. Cette définition dévoile l'importance d'une approche financière par le profit issue de l'ensemble de la chaine logistique. Pour Collin (2005), la SCM est une forme d'organisation aux contours flous basée sur la coopération.

Pour Monnet (2006), la SCM est une forme d'organisation regroupant des acteurs selon un intérêt commun.

La gestion de la chaîne d'approvisionnement est également appelée gestion du réseau d'approvisionnement mondial. Il s'agit d'une approche globale de la logistique qui est axée sur les besoins du client final, pas sur l'héritage des consommateurs, les besoins des fournisseurs, fabricants, grossistes et détaillants. Donc non plus une méthode de gestion séquentielle, mais une intégration de « fournisseurs ». "Le fournisseur du client au client du client "; la gestion de la chaine d'approvisionnement permet une optimisation logistique physique globale ainsi que des flux d'informations de tous les acteurs de la chaine logistique ; par conséquent, nous pensons que la gestion de la chaine d'approvisionnement comme étant un système de gestion d'entreprise étendu. Il permet une large gamme de gestion à travers une fonction de gestion horizontale globale face aux clients de l'amont à l'aval.

La Supply Chain Management (SCM) reflète la compréhension suivante : Les performances de chaque maillon de la chaine logistique sont interdépendantes. Atteindre la performance globale de l'ensemble de la Supply Chain est favorisé à la recherche d'une alternative locale optimale (entreprise interne).

La gestion de la chaine d'approvisionnement (SCM) consiste à superviser l'ensemble du processus des marchandises, des informations et des finances, des fournisseurs aux consommateurs, fabricants, grossistes et détaillants.

La gestion d'approvisionnement implique la coordination et l'intégration de ces processus au sein et entre les entreprises.

L'objectif principal du SCM est d'améliorer la performance de la chaine logistique de l'organisation et de l'ensemble du réseau, cette performance requiert la participation active de tous ses acteurs. Cependant, le SCM demande une collaboration de chaque service avec les autres services de l'ensemble des acteurs de la chaine logistique.

Aussi, le but de tout système SCM est de réduire les stocks (en supposant que les produits soient disponibles à la demande). Pour une gestion réussie de la chaine d'approvisionnement, les logiciels complexes dotés d'une interface Web sont en concurrence avec les fournisseurs de services

\footnotetext{
${ }^{4}$ Ibid, p27.
} 
d'applications Web (ASP). Ce dernier s'engage à fournir tout ou partie du service SCM à l'entreprise qui emploie son service ${ }^{5}$.

\section{L'analyse de l'impact de Covid19 sur l'activité Import-export du Port Tanger Med et ses} répercussions sur la logistique nationale

L'activité économique du Maroc et le reste du monde a été affectée par l'émergence d'une crise sanitaire survenue au cours de l'année 2020 et qui a frappé tous les pays sans exception. Cette crise qui a surpris le monde entier a touché tous les secteurs économiques, sociales sportifs, culturels, etc. C'est un contexte nouveau qui surgit dont les retombées sont néfastes pour l'économie, le social, le citoyen et l'environnement. Face à cette terrible situation, les gouvernements du monde entier se sont retrouvés sous le choc après les pertes humaines enregistrées sans précédent quotidiennement et ont décidé en conséquence de se mobiliser pour limiter la propagation du virus et réduire les dégâts. Cette mobilisation qui s'inscrit dans le cadre de la coopération internationale s'est marquée par la prise d'un ensemble de mesures pour empêcher davantage la propagation du virus. Ces mesures comprennent la quarantaine, l'interdiction et les restrictions de voyage, l'application de la distanciation sociale, la fermeture de lieux publics et l'annulation d'événements publics. En général, il s'agit de la fermeture totale et générale des frontières internationales (maritimes, aériennes et terrestres) de tous les pays face à la libre circulation des personnes, des biens et services. Ces mesures mises en place par les gouvernements avaient pour but de réduire au maximum les effets de la pandémie mondiale sur la santé, la durabilité et le développement économique.

\subsection{La période qui précède l'apparition de la pandémie Covid-19 :}

Au Maroc, depuis le début 2020 et jusqu'à la fin Mars de la même année, les activités du secteur portuaire appartenant au groupe Tanger Med ont connu une hausse par rapport à l'année précédente (30/03/2019). Cette croissance est principalement engendrée par l'augmentation du chiffre d'affaire d'environ 170\% du port Tanger Med lors du premier trimestre 2020. . Ces chiffres sont enregistrés avant l'apparition de la pandémie Covid-19.

Le secteur portuaire s'est révélé comme suit :

-1,3 millions $\mathrm{EVP}^{6}$ de conteneurs, en évolution de 34\% par rapport à la même période de l'année 2019 ;

-107,295 de camions TIR, en croissance de 11\% par rapport à la même période de l'année 2019.

\footnotetext{
${ }^{5}$ www.lemagit.fr.com

${ }^{6}$ EVP : Equivalent Vingt Pieds est une unité qui permet de calculer le volume de conteneurs chargés sur un navire ou stockés dans un terminal.
} 


\subsection{Le port Tanger Med durant la pandémie Covid-19}

Comme toutes les activités économiques qui ont été impactées par la crise sanitaire, le secteur Importexport du port Tanger Med n'a pas subi des conséquences négatives. Son activité n'a pas enregistré des modifications importantes, elle restait presque identique par rapport à la période antérieure de la crise. En effet, en dépit de l'arrêt de l'activité d'un grand nombre important des entreprises marocaines, les services du port restent toujours opérationnels au cours de cette période difficile tels que le stockage, l'entreposage, le remorquage afin de garantir le trafic maritime dans des conditions meilleures conditions meilleures. Le port persiste d'exercer son activité sur la base des critères nouveaux, notamment sanitaires imposées par les autorités marocaines. La situation géographique a été un facteur favorable pour la poursuite du fonctionnement du complexe portuaire durant cette crise. Le climat de confiance créé dans le respect strict des mesures sanitaires a aidé le port d'assurer ses engagements d'approvisionnement avec ses partenaires aussi bien locaux qu'internationaux. Dans ce sens, les chiffres de l'activité transbordement qui représentent plus de 70\% de l'activité globale, sont restés stables par rapport à la période qui a précédé la crise. Pour ce qui est de l'import-export, le rythme n'a pas changé profondément et a continué de fonctionner comme auparavant. Les navires continuent leur trafic, la douane et l'ensemble des départements travaillent de façon normale pour assurer le bon fonctionnement et l'accompagnement de l'économie marocaine durant cette période difficile de la conjoncture mondiale. En termes de valeur, l'activité du port a enregistré 50\% de l'ensemble des échanges extérieurs du Royaume, le reste des autres ports du pays ont occupé la deuxième place.

\subsection{L'analyse du trafic global du Port Tanger Med sous l'effet de la crise sanitaire}

Le complexe portuaire Tanger Med joue un rôle indéniable dans l'organisation et la localisation des activités économiques sur le territoire tangérois. C'est un pôle générateur d'emplois. Sa simple présence est aussi une source d'attraction des entreprises qui par leurs activités, crée également des emplois.

Tanger Med a enregistré une croissance pour l'ensemble des activités portuaires à fin 2020. Cette performance lui a valu la position de premier port à conteneurs sur le bassin méditerranéen. Les derniers chiffres du bilan portuaire, arrêtés à fin décembre 2020 font état de la bonne prestation du complexe Tanger Med.

Selon les chiffres recueillis auprès de l'autorité portuaire de Tanger Med (TMPA) ${ }^{7}$, le tonnage global traité durant l'année écoulée s'est, en effet, élevé à 81 millions de tonnes, en progression de $23 \%$ par rapport à 2019 , notant que le port traite, à lui seul, près de $47 \%$ de l'ensemble du tonnage portuaire du pays.

\footnotetext{
${ }^{7}$ TMPA : Tanger Med Port Authority.
} 
Au niveau national, sur un trafic de 147 Millions de tonnes traités jusqu'à Décembre 2020, les complexes portuaires de Tanger Med, Casablanca et Jorf Lasfar concentrent presque 85,4\% du trafic national. Ainsi, le port de Tanger Med vient en première position et ce grâce à son activité principale de transbordement des conteneurs. Pour le trafic domestique, le port de Jorf Lasfar garde sa première position du pays en termes de volume de marchandises traitées en import-export. Ce résultat enregistré par le Port Tanger Med s'explique par sa situation géographique et sa proximité de l'Europe.

Les principaux trafics des ports marocains concernent les conteneurs, la pêche, les céréales, les véhicules neufs, les phosphates et produits liés, les $\operatorname{TIR}^{8}$, les produits énergétiques, les passagers et les croisières. L'analyse porte sur l'état des lieux de chaque trafic au niveau du port Tanger Med et au niveau national.

\section{Conteneurs}

Sur le plan national, le volume global des conteneurs (import-export) traités par les ports marocains ont atteint, à fin Décembre 2020, un cumul de 1.625.134 conteneurs EVP, soit une baisse de 1,7\% par rapport à la même période de l'année précédente, dont près de $75,7 \%$ est transité par les ports de Casablanca et d'Agadir. Le port Tanger Med vient en deuxième place au niveau du trafic Import/Export derrière le port de Casablanca avec un taux de 5,5\% et occupe la première place au niveau du trafic de transbordement sur le plan national avec un taux de $76,8 \%$.

\section{Céréales}

Sur le plan national, les quantités importés des céréales (Blé, Mais, et Orge) ont connu une augmentation pour atteindre 9,4 millions de tonnes en 2020, contre 7 millions de tonnes de l'année écoulée ( $+35,2 \%)$. Une importante augmentation liée à la forte demande nationale enregistrée depuis la déclaration des mesures de confinement, sous l'effet du Covid19. Pour le port Tanger Med, il n'occupe que 0,9\% du trafic des céréales au niveau national puisque le reste du trafic de cette activité connait une concentration autour des ports de Casablanca (57,3\%) et Jorf Lasfar (21\%).

\section{Phosphates et produits liés}

Sur le plan national, le trafic du phosphate brut et produits liés a atteint, en 2020, un volume global de 37,5 Millions de tonnes, soit une hausse de 13,6\% par rapport à l'année précédente 2019.

\footnotetext{
8 TRI : Transit International Routier est un régime fiscal douanier régie par une convention qui date de 1975 et élaborée sous l'égide de la Commission économique pour l'Europe des Nations-unies (CEE-ONU). Elle établit un système de transit douanier conçu pour faciliter la circulation des marchandises dans le transport international routier.
} 
Les exportations du phosphate brut ont atteint un volume de 10 millions de tonnes, soit une hausse de 4,3\% comparativement à l'année 2019. Sauf pour l'acide phosphorique, tous les autres produits liés ont marqué une variation positive.

Le port Tanger Med semble être à l'écart du trafic des phosphates et des produits liés et exclu du traitement de cette activité aussi importante pour l'économie marocaine.

\section{Les produits énergétiques}

Sur le plan national, le volume des importations hydrocarbures ont enregistré à la fin de l'année 2020, 13,1 Millions de tonnes, marquant ainsi une croissance de 3,2\% par rapport à la fin de l'année 2019. Cette activité a connu une reconfiguration des flux à travers les différents ports de transit avec une progression au niveau des ports de Tanger Med $(+62,4 \%)$ qui arrive dans la première place, Tan-Tan $(+19,1 \%)$ et Dakhla $(+18,6 \%)$ et une baisse au niveau des autres ports : Nador $(-8,3 \%)$, Mohammedia (13\%), Jorf Lasfar (- 16,5\%), Agadir (-2\%) et Layon (-7,5\%).

Pour le trafic des hydrocarbures, le port Tanger Med occupe la deuxième place avec 32,1\% derrière le port de Mohammedia qui arrive en première position avec une contribution de 33,5\%.

\section{Transport International Routier (TIR) :}

A la fin de l'année 2020, le trafic national TIR a atteint un volume de 378.418 unités, dont 95\% est transité par le port Tanger Med, ainsi une augmentation de 1,2\% par rapport à fin Décembre de l'année 2019, en dépit de la crise sanitaire suivi du port de Nador avec 5,02\% et le port de Casablanca avec $0,02 \%$.

\section{Véhicules}

Le trafic des véhicules neufs traité par les ports en 2020, a atteint 425.378 unités contre 589.200 unités de l'année 2019, enregistrant ainsi une diminution de 27,8\%. Ce recul est dû à la suspension temporaire de l'activité des principaux opérateurs au Maroc, suite à la crise sanitaire du Covid-19. Le port Tanger Med a traité 358 milles véhicules neufs en 2020 contre 494 milles en 2019, soit une diminution de 27,4\% par rapport à l'année précédente. Le port Tanger Med accapare 73, $6 \%$ du trafic national des véhicules neufs suivi du port de Casablanca avec un taux de $15,8 \%$.

\section{Pêche côtière et artisanale}

Les débarquements des produits de la pêche côtière et artisanale ont atteint un volume de 1.296.358 tonnes en 2020, dont 98,5\% dans les entrées portuaires atlantiques, soit une baisse de 7,1\% par rapport à l'année précédente 2019. 
En termes de valeur marchande, les produits de la pêche côtière et artisanale débarqués se sont élevés à 6,7 milliards de dirhams en 2020, enregistrant une baisse de 8,5\% comparativement à 2019 .

Les ports atlantiques sont fortement dominants du trafic des produits de mer tandis que le port Tanger Med ne figure plus parmi les ports marocains concernant ce type de trafic.

\section{Passagers et croisiéristes}

Au niveau national, le trafic des passagers ayant transité par les ports marocains, en 2020, a atteint un nombre de 1.003 .931 passagers, soit une diminution de 80,4\% par rapport à l'année précédente, suite aux mesures sanitaires prises par les autorités marocaines pour limiter la propagation du Covid-19, et ce à travers la fermeture des frontières et la suspension de l'ensemble de lignes maritimes entre les ports marocains et les ports étrangers pour le trafic des passagers.

En ce qui concerne le trafic des croisières, les ports marocains ont enregistré 27.339 croisiéristes, soit une baisse de $85,9 \%$ par rapport à l'année précédente. Le port Tanger Med occupe la première place au niveau du trafic des passagers au niveau national avec un taux de 68\% suivi de Tanger ville avec 18,6\% et du Nador avec 10,6\%. Ces chiffres s'expliquent par la proximité du port Tanger Med de l'Europe.

\subsection{Altérations produites par Covid19 sur la chaîne d'approvisionnement nationale}

Alors que l'épidémie de Covid-19 s'étend sur l'ensemble du globe, certains secteurs au Maroc subissent une forte demande des consommateurs et d'autres sont contraints de s'arrêter par décision politique ou à cause de la fragilité de leur chaine logistique.

Au Maroc, comme une économie axée sur la consommation, l'économie a subi des pertes importantes, Ceci sollicite à analyser l'impact de ce virus sur la chaine d'approvisionnement marocaine en se focalisant sur le port Tanger Med.

En effet, cette crise a provoqué des perturbations dans les chaines d'approvisionnement à l'échelle mondiale en raison de la fermeture des frontières maritimes, terrestres et aériennes en Chine et le reste du monde. Cela a eu une influence négative sur tous les secteurs économiques. Il s'agit d'une crise à double face ; une face économique et une face humaine. C'est un double «Choc de l'offre et de la demande» au niveau économique. L'effet de cette conjoncture a été manifestement remarqué à la fois au niveau de la production, la consommation et l'investissement. Cette période difficile est une source d'instabilité et d'incertitude à laquelle le monde devrait y confronter avec courage, détermination et vision.

Au Maroc les entreprises dans le cadre de la mise en œuvre du plan national de veille et de riposte à ce virus, diverses dispositions émanant des notes circulaires du Ministère de la Santé et du Ministère de l'Equipement, du transport de la logistique et de l'eau ont été adoptées dans la gestion du trafic portuaire 
(commerce, passagers, pêche hauturière et plaisance). L'adoption de mesures d'éloignement social, d'auto-isolement et les restrictions de voyage ont entraîné une réduction de la main-d'œuvre dans tous les secteurs économiques et provoqué la perte de nombreux emplois. Le besoin de produits de base et de produits manufacturés a diminué en raison de la réduction du trafic portuaire. En revanche, les besoins en fournitures médicales ont considérablement augmenté. Le secteur alimentaire est également confronté à une demande accrue en raison des achats de panique et de la constitution de stocks de produits alimentaires.

L'arrêt d'activités de plusieurs lignes de production au Maroc fait que les exportations marocaines sont confrontées à une situation unique sans précédent.

Vu l'interdépendance de l'économie marocaine avec l'économie mondiale, le commerce extérieur marocain s'est vu impacté par la propagation du virus et les mesures prises par le gouvernement pour le contenir. Les industriels et les exportateurs voient leur business s'effondre, ce recul dramatique se traduit par des ruptures de stocks, des lignes de production à l'arrêt faute d'intrants, des contrats suspendus, et des déficits de paiement.

Parmi les secteurs qui ont été profondément touché par la pandémie, on trouve que le secteur des médicaments est un secteur exportateur qui a subi une rupture de stocks au niveau du marché africain expliquée par le fait que chaque exportation de médicament est désormais soumise à une autorisation et une étude de cas par cas. Aussi le secteur de la pêche s'est retrouvé touché d'une part par le repos biologique du début de l'année et d'autre part par l'impact négatif du virus sur l'approvisionnement de la matière première en amont, ce qui a eu un impact direct sur les capacités d'exportation.

Quant au secteur de l'agroalimentaire considéré comme un secteur pilier des exportations marocaines, il se stabilise après de nombreuses entraves liées à la baisse de la demande, le retard des livraisons et l'annulation de plusieurs commandes.

\section{Conclusion}

Le Maroc comme le monde entier traverse une période très difficile de l'histoire de l'humanité manifestée par l'apparition et la propagation du coronavirus ou également appelé covid19. Une pandémie qui a créé l'inquiétude, la peur et la panique du globe s'est traduite par des pertes humaines sans précédent et des dégâts économiques jamais connus dans l'histoire contemporaine des nations.

Vu la forte dépendance avec la Chine qui produit $20 \%$ de la richesse mondiale, ce virus a touché l'économie de plusieurs pays. Comme notre pays n'est pas à l'abri de ce qui se passe ailleurs, l'économie nationale a subi des pertes importantes dans tous les secteurs des activités économiques dont les plus touchés par la crise pandémique sont notamment le tourisme, l'automobile et le textile sous l'effet de la fermeture des frontières. La consommation était fortement affectée et la chaine d'approvisionnement 
aussi. En outre, la forte dépendance de l'économie marocaine vis-à-vis de l'Europe, l'économie nationale est affectée par la régression de la croissance parce qu'elle représente plus de $58 \%$ des exportations marocaines, $70 \%$ des revenus du tourisme et $69 \%$ des MRE. L'impact de la crise pandémique sur la chaine d'approvisionnement nationale se confronte au manque de données statistiques officielles suite au confinement. Il était donc très difficile d'analyser les effets de la crise sur chaque secteur à part sans recourir aux méthodes reconnues par la recherche scientifique.

Au niveau du commerce international, c'est toutes les chaines logistiques sont touchées par la propagation du coronavirus et les mesures prises pour lutter contre la pandémie, chose qui s'est répercutée sur le commerce du Maroc avec l'étranger. Les volumes de transport maritime mondial ont baissé suite à l'épidémie de Covid-19.

La crise a révélé que les profonds changements doivent opérer tant dans le comportement des consommateurs que dans la gestion des approvisionnements côté entreprises. Pour s'en sortir au mieux, la digitalisation des procès, la remise en cause des systèmes d'approvisionnement existants et la capacité à s'adapter à de nouveaux modes de consommation qui s'imposent déjà comme des atouts essentiels.

\section{BIBLIOGRAPHIE}

[1] Abbad, H., (2009), « Le choix des PME par la grande distribution alimentaire : quel poids pour les critères logistiques? », Management \& Avenir, 2009/4 n 24.

[2] Adam Ledunois, S. Baudasse, T., Renault, S., (2010), «Proximité et capital social : le cas des Parcs Industriels Fournisseurs », Management \& Avenir, 2010/3 n 33.

[3] Arnaud, N. et El Amrani, R., (2010), «Collaboration électronique et investissement relationnel. Étude de cas exploratoire d'un SIIO dans le secteur du meuble », Revue française de gestion, 2010/8 ${ }^{\circ}$ 207.

[4] BAGLIN (G) : Management industriel et logistique, édition ECONOMICA, Paris, 1996.

[5] Brulhart, F., Claye-Puaux, S., (2009), « Réseau, capital social et performance pour l'organisation : le cas des responsables de sites de prestation logistique », Management \& Avenir, 2009/4 n 24.

[6] Calvi, R., Blanco, E., Koike, T., (2005), « Coopérer en conception pour améliorer les supply chains de demain. Un défi pour les entreprises virtuelles », Revue française de gestion, 2005/3 no 156.

[7] CHRISTOPHER (M) : Supply Chain Management, 3éme édition, édition village mondial, Paris, 2005.

[8] Dang Nguyen, G. et Mevel, O., (2007), « Nouvelle et ancienne économie .Vers une intégration réussie? », Revue française de gestion, 2007/4 n 173 .

[9] Detchessahar, M., Journé, B., Kessous, E., (2003), « Logistique et modèles d'affaires. L'exemple du commerce électronique », Revue française de gestion, 2003/2 no 143.

[10] EYMERY (P) : La logistiques de l'entreprise « Supply Chain Management », édition Hermès, Paris, 1997.

[11] Fabbe-Costes, N. et Lancini, A., (2009), « Gestion inter-organisationnelle des connaissances et gestion des chaînes logistiques : enjeux, limites et défis », Management \& Avenir, 2009/4 n 24. 
[12] Gozé-Bardin, I., (2009), «Les défis de la logistique de distribution à l'horizon 2035 », Management \& Avenir, 2009/4 $\mathrm{n}^{\circ} 24$.

[13] Halley, A., (2004), « Sous-traitance et chaîne logistique ou la nécessaire intégration des deux stratégies », Gestion, 2004/2, Vol. 29.

[14] LAURENTIEN (J) et autres : Processus et méthodes logistique, édition Afnor, Paris, 2000.

[15] MARTIN (C) : Supply Chain Management, village mondial, 3éme édition, Paris, 2005.

[16] MBA, édition d'organisation, Paris, 2005.

[17] POIRIER (C) et autres : Optimiser la chaine logistique et le réseau interentreprises, DUNOD, Paris, 2001.

[18] SHOSHANAH (C) et ROUSSEL (J) : Avantage Supply Chain, Edition d'organisation, Paris, 2005.

[19] TEXIER (D) et autres : La logistique d'entreprise, édition DUNOD, Paris, 1996.

[20] VALLIN (P) : La logistique modèle et méthodes, édition ECONOMICA, Paris, 2006. 